\title{
Psychodrama und gesellschaftlicher Wandel
}

\author{
Falko von Ameln
}

Online publiziert: 5. Oktober 2017

(C) Springer Fachmedien Wiesbaden GmbH 2017

Dass wir in Zeiten des Umbruchs leben, ist offensichtlich. Während ich diese Zeilen schreibe, gedenken die Menschen in Barcelona der Opfer des Terroranschlags vom 17. August 2017. In den USA regiert ein Präsident, der offenbar für Politik weder Interesse noch Gespür aufweist. Das geopolitische Gleichgewicht hat sich mit dem (durch Trump noch beschleunigten) Bedeutungsverlust der USA hin zu einer multipolaren Unübersichtlichkeit verschoben. Die Einheit und Stabilität des europäischen Projekts sind durch Brexit, nur oberflächlich bewältigte Rettungsaktionen für südeuropäische Staaten und Differenzen in der Flüchtlingspolitik in Frage gestellt. Die Schere zwischen Arm und Reich klafft immer weiter auseinander, so dass führende Wirtschaftsvertreter (!) in den USA schon auf eine gerechtere Verteilung des Reichtums drängen. Bei der Bewältigung des Klimawandels gehen viele Staaten nicht über Lippenbekenntnisse hinaus. Die „Diesel-Krise“ hat nicht nur unselige Verflechtungen zwischen Autobranche und Politik offenbart, sondern auch die mangelnde Fähigkeit und/oder Bereitschaft, Zukunftstrends rechtzeitig zu erkennen und mit zukunftsweisenden Entwicklungen aufzugreifen. Die Vernichtung von Regenwald, bedrohten Tierarten, Korallenriffen usw. schreitet in bedrohlichem Tempo fort. All diese Fehlentwicklungen geschehen in einer Zeit, die über ein nie dagewesenes Wissen über ökologische Zusammenhänge verfügt, über (zumindest in den deutschsprachigen Ländern) einen nie dagewesenen Wohlstand zur Bewältigung sozialer Probleme, ein nie dagewesenes Bildungsniveau und ein Niveau der demokratischen Entwicklung, das niemals höher war als heute.

Natürlich haben Veränderungen zu jeder Zeit Verunsicherung mit sich gebracht und zu jeder Zeit haben Menschen im Alter der in diesem Band versammelten AutorInnen (einschließlich des Herausgebers) im Angesicht des Wandels einen Wer-

F. von Ameln $(\bowtie)$

Norden, Deutschland

E-Mail: info@vonameln.net 
teverlust beklagt. Ebenso ist natürlich morgen auch nicht alles radikal anders, geschweige denn notwendigerweise schlechter als gestern.

Dennoch ist es sicher nicht übertrieben, von epochalen Veränderungen zu sprechen. In der Geschichte der Menschheit hat jede Etablierung eines neuen Kommunikationsmediums radikale Veränderungen ausgelöst. Dies gilt für die Entwicklung der Sprache, die Erfindung der Schrift oder des Buchdrucks und der Telegrafie ebenso wie für die heute rapide fortschreitende Digitalisierung. „Wir haben es“, so Dirk Baecker (2007, S. 7),

mit nichts Geringerem zu tun als mit der Vermutung, dass die Einführung des Computers für die Gesellschaft ebenso dramatische Folgen hat wie zuvor nur die Einführung der Sprache, der Schrift und des Buchdrucks. Die Einführung der Sprache konstituierte die Stammesgesellschaft, die Einführung der Schrift die antike Hochkultur, die Einführung des Buchdrucks die moderne Gesellschaft und die Einführung des Computers die nächste Gesellschaft. Jedes neue Verbreitungsmedium konfrontiert die Gesellschaft mit neuen und überschüssigen Möglichkeiten der Kommunikation, für deren selektive Handhabung die bisherige Struktur und Kultur der Gesellschaft nicht ausreichen. Jede Einführung eines neuen Verbreitungsmediums muss daher zur Umstellung dieser Struktur führen ...

Vor diesem Hintergrund wird erkennbar, dass wir derzeit Erschütterungen erleben, die nicht nur Ökonomie, Ökologie und Geopolitik betreffen, sondern die Umwälzungen in der Sozialstruktur bewirken, die bis ins Private, ja Intrapsychische hineinreichen und die heute erst im Ansatz spürbar und verstanden sind. Gerade für uns PsychodramatikerInnen, die wir gelernt haben, dass das vermeintlich Individuelle immer ein Spiegel der sozialen Verhältnisse ist und dass der Mensch nie abgelöst von seinem sozialen Kontext verstanden werden kann, sollte dieser Befund eine Verpflichtung sein, die gesellschaftlichen Veränderungen unserer Zeit kritisch zu beobachten und zu begleiten.

Dem Psychodrama ist ein gesellschaftspolitischer Anspruch immanent - Moreno hat seine Überzeugung, dass wir alle in einem weltumspannenden Netzwerk sozialer Beziehungen (womit er nicht Facebook meinte), z. B. mit seinem Einsatz für Flüchtlinge eindrucksvoll in Handeln umgesetzt. Psychodrama ist niemals nur auf den einzelnen Klienten oder die einzelne Klientin bezogen, sondern immer ,,social atom repair work“", das auf eine Verbesserung des Zusammenlebens abzielt. Dieser von Moreno propagierte sozialtherapeutische Anspruch, auf den vor allem Ferdinand Buer mit seinem unermüdlichen Schaffen immer wieder hingewiesen hat, ist heute wichtiger denn je. Wahrscheinlich werden die PsychodramatikerInnen auch Donald Trump und Kim Jong Un nicht zum Rollentausch bewegen können, dennoch darf das Psychodrama nicht zur unpolitischen Sozialtechnologie verkommen, die gesellschaftliche Problemlagen reproduziert, statt sie in Frage zu stellen.

In dieser Situation versteht sich der vorliegende Sonderband der Zeitschrift für Psychodrama und Soziometrie als Hilfe zu einer Positionsbestimmung. Wir haben eine Reihe von AutorInnen gebeten, zu aktuellen gesellschaftlichen Sorgethemen Stellung zu nehmen. Der Anspruch bestand dabei darin, Entwicklungen in diesen Themenfeldern auf einer „Flughöhe“ der Beobachtung zu thematisieren, die 
der Komplexität des Gegenstandes angemessen ist und die relevanten gesellschaftspolitischen und wissenschaftlichen Diskurse berücksichtigt. Herausgekommen sind fundierte Analysen ebenso wie ganz persönliche Standpunkte, die zum Nachdenken und zur Reflexion der eigenen Haltung als PsychodramatikerInnen einladen sollen.

Das zweite Ziel unseres Projekts, nämlich die theoretisch-analytischen Erörterungen auf ihre Implikationen für die psychodramatische Arbeit hin auszuloten, steht eher im Hintergrund. Einer ebenfalls von Moreno ausgehenden, aber weniger rühmlichen Traditionslinie folgend, neigt das Psychodrama gelegentlich dazu, komplexe Problemlagen auf einfache Lösungsvorschläge engzuführen. Auch wenn psychodramatische Arbeit sicherlich an vielen Stellen etwas bewegen kann, werden sich Klimawandel und Kriege nicht durch einen Rollenwechsel abstellen lassen. Wenn die Beiträge die LeserInnen daher bisweilen mit offenen Fragen zurücklassen, ist dies durchaus beabsichtigt.

In seinem Eröffnungsbeitrag spannt Ferdinand Buer gewissermaßen den thematischen Rahmen für die folgenden Beiträge auf. Anhand eines kurzen Abrisses von Morenos Schaffen und indem er Morenos Werk mit dem anderer Soziologen und Philosophen in Beziehung setzt, legt Buer dar, dass im Psychodrama Diagnose und Intervention ineinander verschränkte Dimensionen darstellen. Auf dieser Basis erörtert Buer, wie das Psychodrama zur Verbesserung der Lebensqualität und zur Förderung von „Konvivialität“ beitragen kann.

Thomas Schwinger und Christoph Hutter befassen sich mit den Veränderungen, die derzeit in Beziehungen im Allgemeinen sowie in Familien zu beobachten sind. Beide Autoren präsentieren eine sehr eingehende und soziologisch fundierte Analyse, die viele Denkanstöße beinhaltet.

Katharina Novys Beitrag lädt zu einer intensiveren Beschäftigung mit der Demokratie ein, die für viele als selbstverständlich gilt (aber in gewisser Weise immer mehr an Selbstverständlichkeit verliert). Novy belegt, wie rechtspopulistische Parteien versuchen, die Demokratie mithilfe von drei Strategien zu unterhöhlen: die Konstruktion eines „Wir“ in Abgrenzung von „den Anderen“, Verweigerung und Unterminierung von Diskursen sowie das Schüren von Verunsicherung und Ängsten. Vor diesem Hintergrund zeigt die Autorin auf, wie das Psychodrama zur Wahrung und Weiterentwicklung von Demokratie beitragen kann.

Konrad Schnabel und Janna M. Gottwald beleuchten die aktuellen Migrationsbewegungen, wobei vor allem die Faktoren in den Blick genommen werden, die es MigrantInnen schwer machen, hierzulande Fuß zu fassen. In ihrem sehr praxisbezogenen Artikel stellen sie dar, wie Psychodrama und Soziodrama genutzt werden können, um die mit Migration verbundenen Herausforderungen besser zu bewältigen.

Wolfgang Dietrich, Inhaber des UNESCO-Lehrstuhls für Friedensforschung, setzt die in den letzten Jahrhunderten zu beobachtenden Veränderungen in den Konfliktbildern in Beziehung mit der Entwicklung der Friedensforschung und der Humanistischen Psychologie. Dietrich greift die Unterscheidung von präskriptiver und elicitiver Konfliktlösung auf (,Präskriptive Konfliktlösung trifft Menschen in der künstlichen Umgebung von Konferenzräumen. Elicitive Konflikttransformation begegnet ihnen auf der Straße und in ihren Heimen, in ihrer natürlichen Umgebung. Präskriptive Konfliktlösung analysiert, diagnostiziert und beurteilt sie fragmenta- 
risch. Elicitive Konflikttransformation lässt sie ihre konflikthaften Rollen ausagieren und hilft, alternative Optionen des Handels und Beziehens zu erkunden“) und rekonstruiert das Psychodrama als elicitive Konfliktlösungsstrategie, die in der aktuellen Situation an Bedeutung gewinnt.

Christian Stadler widmet sich in seinem Beitrag „Nachhaltigkeit als psychologische Herausforderung“ einem zentralen, aber dennoch weithin vernachlässigten Zukunftsthema. Stadler differenziert zunächst den inflationär verwendeten Nachhaltigkeitsbegriff und schildert anhand einer eindringlichen Zeitdiagnose, warum Nachhaltigkeit heute wichtiger denn je ist. Sein Artikel zeigt, dass wir vor der Entscheidung stehen, Nachhaltigkeit auf global Ebene konsequenter zu fördern (,Change by Design“) oder einen „Change by Disaster“ in Kauf zu nehmen. Schließlich zeigt Stadler auf, was das Psychodrama zu einer solchen globalen Nachhaltigkeitsperspektive beitragen könnte.

Christian Pajek greift mit der Veränderung der Medienlandschaft ein weiteres hochaktuelles Thema auf. Seine Überlegungen zeigen, warum wir als PsychodramatikerInnen zu den durch diesen Wandel angestoßenen gesellschaftlichen Veränderungen viel zu sagen haben.

Michael Döllers Beitrag „Selbstoptimierung als Akt der Selbstunterwerfung“ eröffnet den Teil des Sonderbands, der sich intensiver mit den verschiedenen Formaten der Beratung und Therapie auseinandersetzt. In seinem eindringlichen Diskussionsbeitrag wird die Tendenz zur Selbstoptimierung als Akt der Selbstunterwerfung unter die Gesetzmäßigkeiten einer post-demokratischen Marktgesellschaft rekonstruiert. Döller sieht psychodramatische Beratung im Hinblick auf diese Entwicklung in der Rolle des Störenfrieds - eine kritische Stellungnahme, die zu einer eigenen Positionsbestimmung einlädt.

Katja Russo und Bettina Zehetner diskutieren die Folgen der aktuellen gesellschaftlichen Veränderungen für die psychotherapeutische Arbeit. Da, so ihre These, psychisches Leiden immer einen Spiegel gesellschaftlicher Verhältnisse darstellt, haben psychosoziale Beratung und Psychotherapie den Auftrag, die Zusammenhänge zwischen gesellschaftlichen Lebensbedingungen und individuellem Leiden aufzeigen. Wenn sie darauf hinweisen, dass Psychotherapie immer im Spannungsfeld von Ermächtigung und Normierung agiert, greifen sie die in Döllers Beitrag bereits angeschnittene Frage auf, inwieweit psychodramatische Praxis zu einer Veränderung gesellschaftlicher Verhältnisse beitragen kann oder - schlimmstenfalls - nur zu einer Affirmation dieser Verhältnisse beiträgt. Ein besonderer Fokus des Artikels liegt auf Gender-bezogenen Fragestellungen.

Sebastian Verbeek und Eckhard Frick widmen sich einem besonderen Aspekt an der Schnittstelle von gesellschaftlichen Veränderungen und psychotherapeutischer Arbeit, nämlich dem heute zunehmend zu beobachtenden ,narzisstischen Hunger“ nach Selbstinszenierung. Die Autoren unterscheiden verschiedene Erscheinungsformen des Narzissmus und geben auf dieser Basis Empfehlungen für die psychodramatische Praxis.

Klaudia Niepenberg beschreibt den mit ökonomischen Zwängen gepaarten Leistungsdruck (,effektiv, hochwertig, kostengünstig“) in der sozialen Arbeit, hinter dem Werte wie Menschlichkeit und Solidarität zurückzutreten drohen. Das Psychodrama 
kann, so ihre These, dazu beitragen, die gewachsenen Konserven der sozialen Arbeit zu durchbrechen und diesen Werten wieder zu stärkerer Geltung zu verhelfen.

Michael Döller greift die aktuellen Veränderungen in der Arbeitswelt und das in der Organisationswelt zurzeit vieldiskutierte Leitbild der agilen Organisation auf. Von seiner sehr anschaulichen Beschreibung der Lage ausgehend weist Döller auf die Paradoxien hin, die die aktuelle Situation prägen. Das Psychodrama sieht er als Ansatz für eine Entwicklung hin zu einer echten Partnerschaftlichkeit zwischen UnternehmerInnen, Führungskräften und MitarbeiterInnen, die diese Paradoxien abmildern könnte.

Matthias Spörrle, Thomas Schneidhofer und Linda Hillinger analysieren die Auswirkungen des gesellschaftlichen Wandels auf das Arbeitsfeld Coaching. Ihr unkonventioneller Blick auf das Thema umfasst dabei auch in den Coaching-Diskursen weniger beachtete Aspekte.

Das letzte Wort hat wiederum Ferdinand Buer, der mit seinem Text „Lebensqualität heute. Was Hartmut Rosa und J. L. Moreno uns dazu sagen können“ ein Thema seines Eröffnungsbeitrages aufgreift und vertieft und damit den thematischen Bogen dieses Sonderbands beschließt.

Die Redaktion der ZPS wünscht eine anregende Lektüre mit zahlreichen Erkenntnissen über eine Zeit im Umbruch!

Falko von Ameln

\section{Literatur}

Baecker, D. (2007). Studien zur nächsten Gesellschaft. Frankfurt/M.: Suhrkamp.

Priv.-Doz. Dr. Falko von Ameln ist Organisationsberater mit Schwerpunkten Change Management und Führungskräfteentwicklung. Er ist an verschiedenen Universitäten und Instituten in der Ausbildung von Berater/innen engagiert. Habilitation im Fach Beratungswissenschaft, Redaktionsmitglied der ZPS und Editor-in-Chief der Zeitschrift „Gruppe. Interaktion. Organisation. (GIO)““. 